\title{
Prediction of Yield of solar modules as a function of technological and climatic parameters
}

\author{
B. Verhelst, D. Caes, L. Vandevelde, Senior Member IEEE, J. Desmet, Senior Member IEEE
}

\begin{abstract}
A solar module is exposed to numerous factors which influence the total yield. Besides the yield being dependent on both the installed technology and placement conditions, some parameters have a direct effect, others have an indirect influence on the actual output. In this study the yield is predicted as a function of both technological and climatic factors. To evaluate the impact of a single parameter, data of a photovoltaic test field with four different technologies and three different test set ups is analyzed to distract an equation for the instantaneous yield of a string of solar modules. An expression is derived to determine the yield based on the ambient temperature instead of the much used panel temperature. This study does not calculate the total yield of the PV system nor does it take into account invertor efficiencies. The yield is calculated at $\mathrm{DC}$ side.
\end{abstract}

Index Terms - Monitoring, Performance Analysis, Photovoltaic Systems, Solar Energy

\section{INTRODUCTION}

The yield of a solar module depends on numerous factors, both technological and environmental [6]. The technological parameters such as efficiency, area per kilowatt-peak $\left(\mathrm{kW}_{\mathrm{p}}\right)$, temperature coefficients on Maximum Power Point (MPP) Power, short circuit current and open circuit voltage are typical for each technology. There are numerous climatic parameters that have a direct or indirect influence on the yield of a solar module. The ambient temperature and the irradiation are the most important factors on the module temperature [1], [2], [8]. Next to these important factors wind speed, pollution, degradation, shadow and humidity have a smaller influence on the yield of the panel [4], [5], [6].

In literature [3] expressions can be found to predict solar yield based on several external parameters. These use the panel temperature as one of the parameters. In the major part of the installations panel temperature is not measured what makes it impossible to use in solar prediction formulas.

This study aims to elaborate a formula who uses the ambient temperature as a parameter for prediction of panel

B. Verhelst, J. J. Desmet and D. Caes are with Technical University Howest, Graaf Karel de Goedelaan 34, Kortrijk, Belgium and associated with Ghent university (e-mail: bart.verhelst@howest.be, jan.desmet@howest.be and david.caes@howest.be).

L. Vandevelde is with Ghent university, Department EESA, SintPietersnieuwstraat 41, Ghent, Belgium (e-mail: lieven.vandevelde@ugent.be). temperature because ambient temperature measurements are widely available. This study will evaluate the gathered data to obtain an equation that estimates the instantaneous yield as a function of the logged parameters to verify the accuracy when using ambient temperature as a parameter. The calculated yield is the yield at the DC-terminals, consequently the efficiency of the inverter is not taken into account. The accuracy of the proposed equation will be tested by comparison between calculated yield and measured data. Based on this equation the impact of each parameter on the yield is determined. With this knowledge, the impact of the most important factors can be optimized in order to maximize the yield for different technologies.

For this study, five minute data over more than one year is available from a photovoltaic test installation with four different technologies, namely polycrystalline, monocrystalline, micro amorphous silicon and monomorphic solar modules. Monomorphic is a compound name for a solar cell who is made of a thin monocrystalline silicon wafer surrounded by ultra-thin amorphous silicon layers (HIT, Heterojunction with Intrinsic Thin layer). These technologies are installed in two setups. The first is an open mounting structure to simulate an installation on a flat roof. The second is a closed mounting structure to simulate an installation on a sloped roof. Polycrystalline and micro amorphous modules are also placed on a double tracking system. In total there are ten strings of solar modules of approximately 1 kilowatt-peak. Each string is connected to an identical inverter.

\section{METHOD}

\section{A. Introduction}

Monthly data has been analyzed for a total period of one year, starting from September 2010 up to August 2011. All results are specified by month. For each setup, module temperature $\left({ }^{\circ} \mathrm{C}\right)$, generated $\mathrm{DC}$ power $(\mathrm{W})$, date, time, ambient temperature $\left({ }^{\circ} \mathrm{C}\right)$, wind speed $(\mathrm{m} / \mathrm{s})$ and irradiation $\left(\mathrm{W} / \mathrm{m}^{2}\right)$ of both the fixed and tracker setup are logged approximately every five minutes. In order to compare the different technologies, all power values are referenced to $1 \mathrm{~kW}_{\mathrm{p}}$. In order to predict future yield, there is need for an equation which takes into account the climate factors which are logged and technological factors from the datasheets. 
The graphs included are from one technology in one setup for one month, especially polycrystalline solar panels in open structure from September. The averaged values are calculated over the whole period.

\section{B. Prediction module temperature}

The module temperature is an important parameter. First, an equation is determined to predict the module temperature as a function of the logged climatic parameters. This equation is then used in the final formula of the instantaneous yield.

Equation (1) gives the expression to predict the temperature of a panel.

$$
T_{\text {pan }}=x \cdot T_{a m b}+y \cdot E-z \cdot V
$$

Where $\mathrm{T}_{\mathrm{amb}}$ is the ambient temperature, $\mathrm{E}$ is the irradiation and $\mathrm{V}$ is the wind speed. Parameters $x, y, z$ express the influence of each parameter. These parameters are determined based on the yield data. The temperature values are converted to Kelvin to avoid problems with negative temperatures during winter. The module temperature is slightly lower than the ambient temperature this due to the position of the temperature sensor. So in summer, the impact factor $\mathrm{x}$ is slightly lower than 1 and inverse during winter because of the negative values of temperature.

In Fig. 1 is explained why the conversion to Kelvin is necessary. In equation (1), the module temperature is expressed as a function of the ambient temperature. Assume factor $\mathrm{x}$ having a value of 0.9 , in summer the expression gives no problems to predict module temperature. In winter factor $\mathrm{x}$ gives wrong results for the module temperature, as can be seen on the figure.

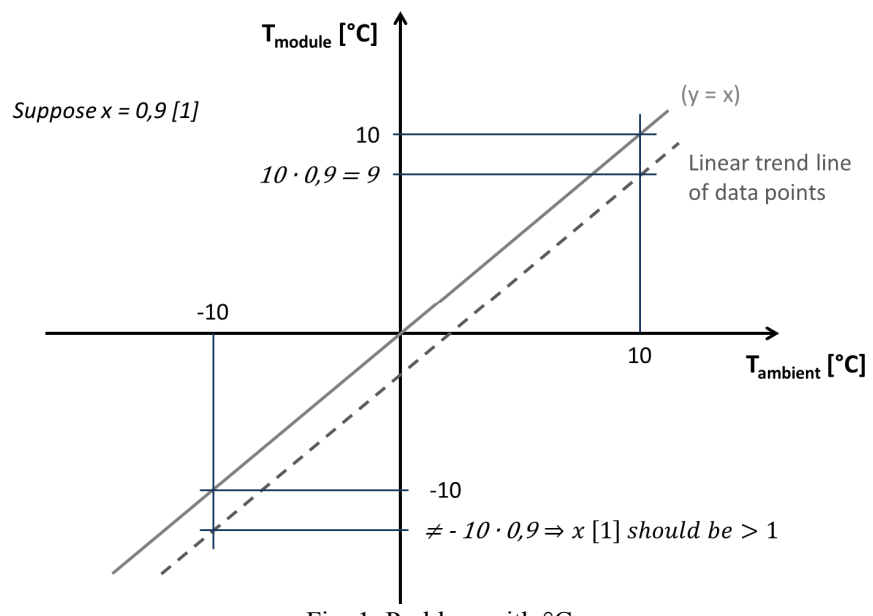

Fig. 1. Problem with ${ }^{\circ} \mathrm{C}$

To determine the impact of each parameter, a selection of the data is made to exclude the influence of other parameters as much as possible.

\section{1) Impact of the ambient temperature}

The selected data to determine the impact of the ambient temperature are only moments without irradiation. From this first selection, moments where module temperature is higher than ambient temperature are also unusable because the module is heating up the environment at this moment.

From the selected data, the relation between the ambient and module temperature is expressed in a graph where the temperature of the panel is set as a function of the ambient temperature. A trend line through the points is the best representation for all data. The correlation coefficients of two different trend lines were evaluated. The linear regression gives similar results for correlation coefficients in comparison with the polynomial trend line. (Fig. 2)

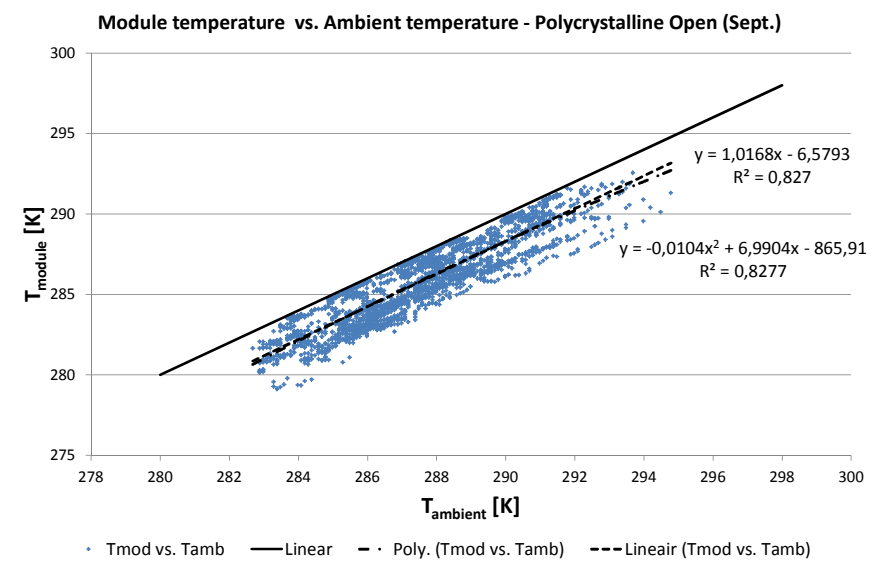

Fig. 2. Impact module temperature

The full black line on Fig. 3 is a linear function with equation $y=x$, this is the border of the module temperature under the influence of the ambient temperature. Without other factors, the module can't adopt a higher temperature than the ambient temperature. To calculate the impact of the ambient temperature on the module temperature, first the minimum and maximum ambient temperature are determined out of the data selection, these are respectively numbers 1 and 2 on Fig. 3 . The expression of the trend line calculates the corresponding module temperature, numbers 3 and 4 . The impact is then calculated as the ratio of 5 to 6 , which are respectively the averages of 1,2 and 3,4 .

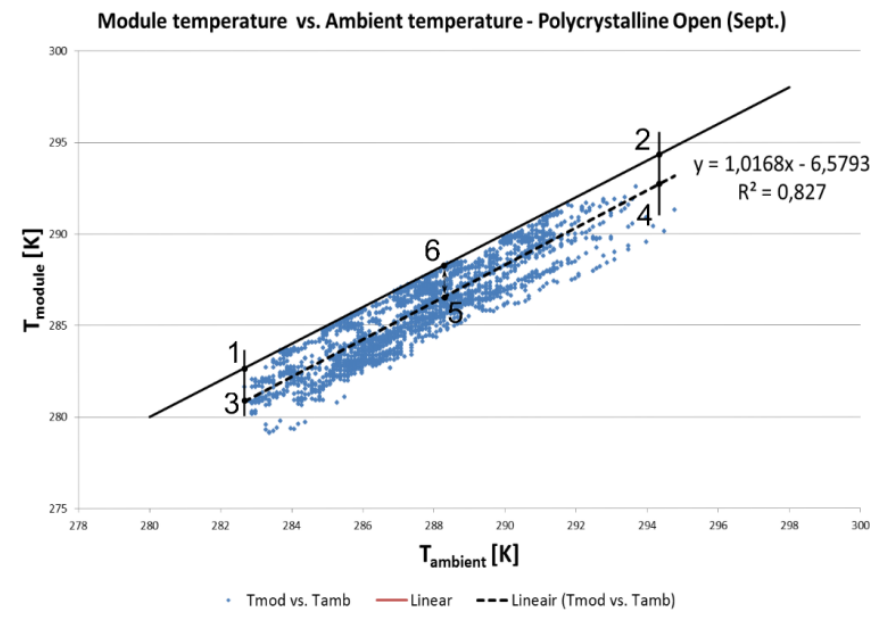

Fig. 3. Determination factor ambient temperature 
The impact of the ambient temperature gives in general values slightly larger than 0.99 . Further in the study this value is rounded to 1 . By rounding, the fault made is less than $1 \%$. This is comparative with an equation found in literature (2) [1]. The impact of ambient temperature is also 1 .

$$
T_{\text {cell }}=T_{a m b}+\frac{N O C T-20}{800} \cdot E
$$

Because this formula uses a technological factor (Nominal Operating Cell Temperature) to describe the impact of irradiation, it will also be used in the final formula to compare the results with the determined formula (1).

\section{2) Impact of irradiation}

The impact of irradiation on the module temperature is determined with another selection of data, only moments when power is produced are selected. To partly exclude the impact of the ambient temperature, the data is used in intervals of ambient temperature. For intervals of $5 \mathrm{~K}$, panel temperature is plotted as a function of irradiation (Fig. 4). A linear and a polynomial trend line are evaluated, but the difference in correlation is small enough to choose the simpler linear expression. Only the slope of the trend line is important, this characterizes the impact of irradiation [7]. The parameter 294,5 of the linear regression expression is the ambient temperature, which provides a base temperature of the panel. Finally, the average of the parameter determining the slope per interval determines the final parameter of the slope for that month.

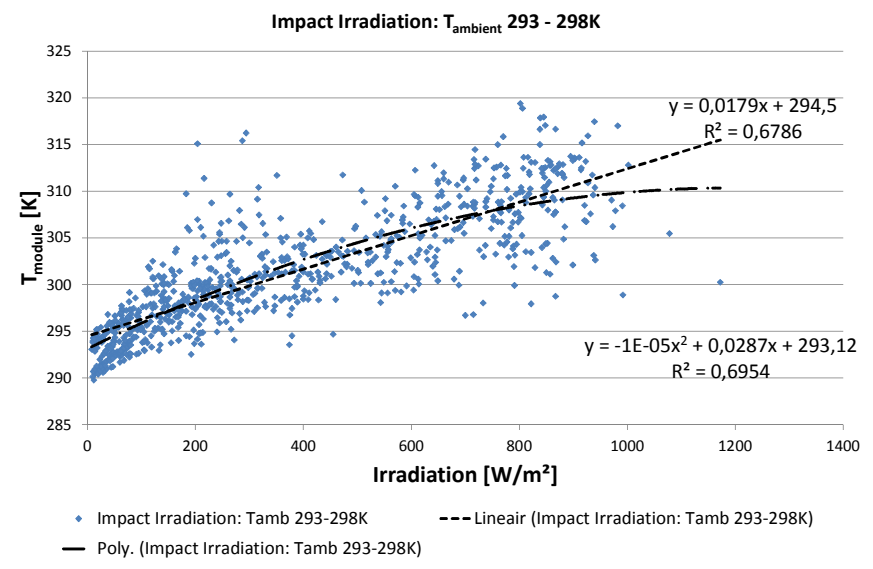

Fig. 4. Impact irradiation

\section{3) Impact wind speed}

The impact of wind speed is difficult to determine because this is a non-seasonal parameter. Because of that, all data of the year is used at once to determine the impact. The selection consists of moments when the ambient temperature is between 25 and $30{ }^{\circ} \mathrm{C}$ and the irradiation between 900 and $1000 \mathrm{~W} / \mathrm{m}^{2}$. These conditions are to be considered warm so the impact of wind speed would be clear.
Fig. 5 shows the relation between the wind speed and the module temperature for the selected moments.

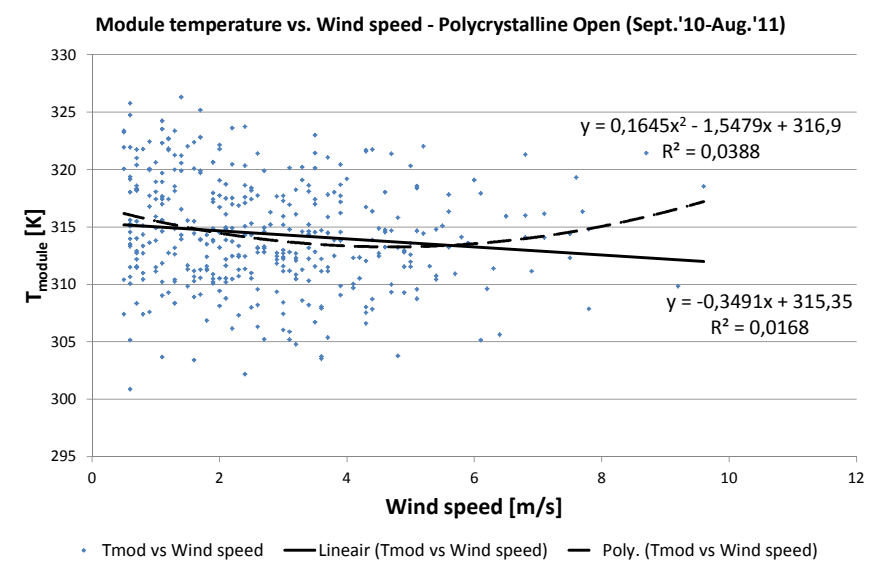

Fig. 5. Determination factor wind speed

No correlation is found in the plot. In [4] and [5] some values are suggested to express the wind impact. In this study a value of $1.45{ }^{\circ} \mathrm{C} /[\mathrm{m} / \mathrm{s}]$ is used to express the impact of wind speed what means that the module cools down $1.45^{\circ} \mathrm{C}$ per meter per second wind speed. In other literature [9] there are more detailed formulas, but useless here because the wind speed is taken into account in the irradiance factor.

There are more factors with influence such as wind direction and air humidity [8] but are not taken into account in this study because this data is not available.

\section{4) Module temperature prediction}

The accuracy of equation (1) is evaluated by comparing the predicted values with the actual logged values, both with and without wind impact. The formula where the impact for wind speed is included gives less good results, so this factor can't be used in this equation. In further evaluation the wind speed is not taken into account.

The actual values without wind are plotted as a function of the predicted values on Fig. 6. Three trend lines are evaluated on correlation to obtain the best expression for all values. The linear regression through the origin, for the formula without wind impact, the correlation coefficient gives comparative results for the three types of trendlines. Because the slope of the linear trend line is almost equal to 1 (0.9964 on Fig. 6), no extra parameters are needed.

The formula (2) with the technological parameter NOCT is also evaluated in the same manner. The correlation for the trend line through the origin is lower than the own formula. This means that the expression is less accurate but can still be used in the final equation for the power because of the simplicity. In case that no climatic data is available, this is a good alternative for yield predictions. 


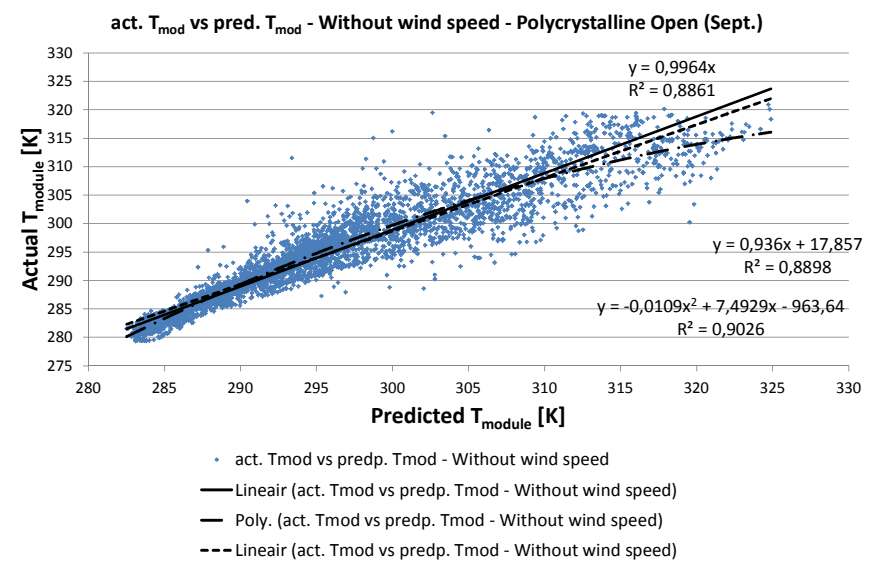

Fig. 6. Actual $\mathrm{T}_{\text {module }}$ vs. predicted $\mathrm{T}_{\text {module }}$

\section{Prediction instantaneous yield}

To predict the instantaneous DC power (P) of solar modules, an equation was made and confirmed in literature [3]. Equation (3) gives the expression with the used factors.

$$
P=\eta \cdot A_{k W p} \cdot(\tau \alpha) \cdot E \cdot\left(1-\gamma \cdot\left[T_{p a n}-25\right]\right)
$$

Where $\eta$ is the efficiency, $A_{k W p}$ is the area per $k_{p}, \tau \alpha$ is the transmission $(\tau)$ - absorption $(\alpha)$ factor and $\gamma$ is the temperature coefficient of MPP Power. The factor $T_{\text {pan }}$ is replaced by the equations as mentioned before (1) and (2). The evaluation of this formula is done in the same manner as the formula for module temperature. The technological parameters are included in the datasheets, except the transmissionabsorption factor. This factor is 0.9 for the fixed setups, 1 for the tracker.
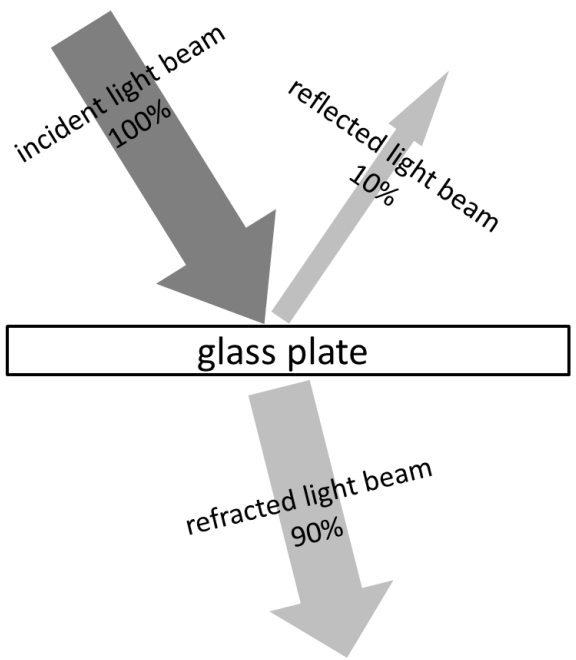

Fig. 7. Transmission-absorption factor

The transmission-absorption factor is a constantly varying parameter for the fixed setups. This is due to the position of the sun. Because irradiation doesn't reach the panel at an angle of ninety degrees, the light is broken by the protection glass plate of the solar panel (Fig. 7). This refraction causes reflection of light. Also the solar cells doesn't capture all the incoming light. Because of no information of this factor, it has a fixed value of 0.9 . The tracker captures the light at an angle of ninety degrees, consequently there's no reflection of sunlight, so the factor is 1 .

The accuracy of the values calculated with formula (3) is verified in the same manner as for module temperature (Fig. 8). Again, three different trend lines are evaluated. This control is done for each month, for each technology and for each setup. The correlation of each trend line are almost equal to each other. The correlation for these predictions is averaged 0.94 , which is certainly acceptable.

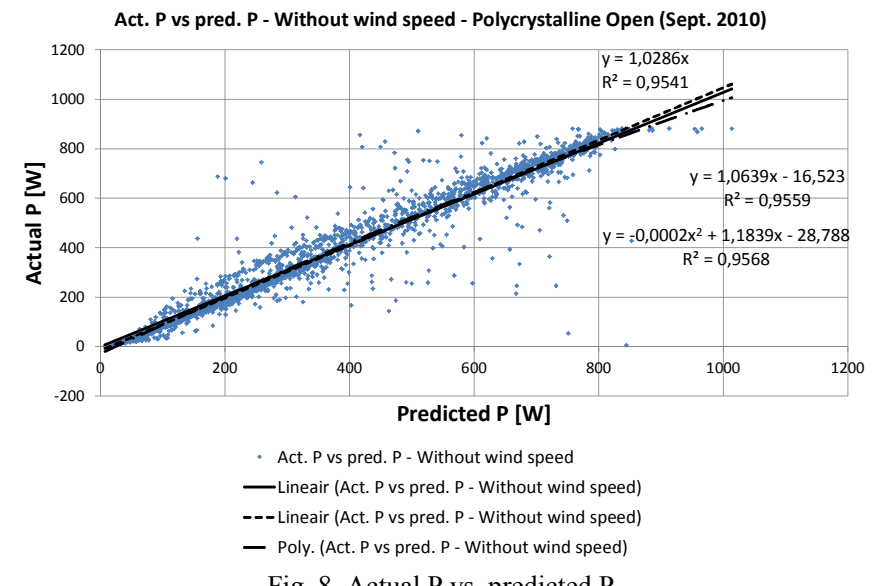

To evaluate the precision, the slope of the linear trend line through the origin is important. The closer the slope is equal to 1 , the more the formula is accurate. To make conclusions for a technology in a certain setup, the average of the entire period, except December, is calculated. The results of December are neglected because of the numerous snow days.

When combining the formula for temperature influence (1) (without wind factor) and instantaneous DC yield (3) following expression can be derived.

$$
\begin{aligned}
& P=\eta \cdot A_{k W p} \cdot(\tau \alpha) \cdot E \\
& \quad \cdot\left(1-\gamma \cdot\left[\left\langle x \cdot T_{a m b}+y \cdot E\right\rangle-25\right]\right)
\end{aligned}
$$

\section{RESULTS}

\section{A. Prediction module temperature}

Using the method described above, the slopes of the linear trend lines through the origin are important to evaluate the accuracy of the formula predicting the module temperature. For the factors $x, y, z$ of equation (1) $\mathrm{x}=1$, y is varying per month and $\mathrm{z}=0$ due to lack of accurate wind data.

Table 1 includes the average slopes of all trend lines from the different technologies and setups over one year. These can be rounded to one if standard deviations are small which is the case. 
Table 1: Averaged slope of trend line by prediction

\begin{tabular}{|l|c|c|c|}
\hline a-T $_{\text {ww }}$ & Closed & Open & Tracker \\
\hline & \multicolumn{3}{|c|}{ Monomorphic } \\
\hline Av. & 1.000 & 1.002 & $/$ \\
\hline Stdev & 0.002 & 0.009 & $/$ \\
\hline & \multicolumn{3}{|c|}{ Micro amorphic } \\
\hline Av. & 0.999 & 0.994 & 0.994 \\
\hline Stdev & 0.005 & 0.005 & 0.004 \\
\hline \multicolumn{3}{|c|}{ Monocrystalline } \\
\hline Av. & 0.998 & 0.994 & $/$ \\
\hline Stdev & 0.004 & 0.005 & $/$ \\
\hline & \multicolumn{3}{|c|}{ Polycrystalline } \\
\hline Av. & 0.995 & 0.993 & 0.995 \\
\hline Stdev & 0.005 & 0.005 & 0.005 \\
\hline
\end{tabular}

Where $a-T_{w w}$ is the slope of the linear trend line through the origin for the prediction of the module temperature at a certain moment. Av is the abbreviation for average and Stdev means standard deviation.

\section{B. Prediction DC power}

As previously explained the linear trend line through the origin gives acceptable results in comparison with other trend lines. The slope of this line is an important parameter to evaluate the accuracy of the formula.

In Table 2 the averaged slopes of the evaluation of the formula (4) to predict the instantaneous power are included. These are averages of the slopes per month from the whole period, except December.

Table 2: Averaged slope of trend line by prediction Power

\begin{tabular}{|l|c|c|c|}
\hline a-P $_{\text {ww }}$ & Closed & Open & Tracker \\
\hline & \multicolumn{3}{|c|}{ Monomorphic } \\
\hline Av. & 1.008 & 1.049 & $/$ \\
\hline Stdev & 0.046 & 0.063 & $/$ \\
\hline & \multicolumn{3}{|c|}{ Micro amorphic } \\
\hline Av. & 0.910 & 0.908 & 0.842 \\
\hline Stdev & 0.036 & 0.032 & 0.022 \\
\hline & \multicolumn{3}{|c|}{ Monocrystalline } \\
\hline Av. & 0.988 & 1.016 & $/$ \\
\hline Stdev & 0.044 & 0.060 & $/$ \\
\hline & \multicolumn{3}{|c|}{ Polycrystalline } \\
\hline Av. & 1.012 & 1.039 & 1.004 \\
\hline Stdev & 0.044 & 0.051 & 0.058 \\
\hline
\end{tabular}

Where a- $\mathrm{P}_{\mathrm{ww}}$ is the slope of the linear trend line through the origin for the prediction of the instantaneous power from the formula without wind impact.

From Table 2 it can be derived that only the micro amorphous technology gives results that deviate more than five percent from 1. All other technologies give slopes within a fault margin of five percent, which is considered as acceptable. The standard deviation are small, so the results from each month of the year are comparable to each other.
For the micro amorphous technology, the average deviation for the fixed setup is about $9 \%$. So the predicted values are about $9 \%$ too high in comparison with the actual values. For the tracker setup, the predicted values for the amorphous technology are about $15 \%$ too high. This difference is caused by a different sensitivity of the metering sensor and panel.

\section{Sensitivity analysis}

The results indicate that the formula is a good manner to predict the DC-power of solar modules, taking into account some technological and climatic factors. Based on this formula, the impact of each parameter can be theoretically determined. Each parameter has a constant value while one parameter is variable. In this way, the relation between the factor and the DC-power can be declared in general.

Table 3 contains the constant values of all parameters of equation (4) for the analysis.

Table 3: Constant values parameters

\begin{tabular}{|l|c|c|}
\hline Factor & Value & Unit \\
\hline$\eta$ & 0.15 & \\
\hline $\mathrm{A}_{\mathrm{kWp}}$ & 10 & $\mathrm{~m}^{2} / \mathrm{kW}_{\mathrm{p}}$ \\
\hline $\mathrm{E}$ & 135 & $\mathrm{~W} / \mathrm{m}^{2}$ \\
\hline$\tau \alpha$ & 0.9 & \\
\hline$\gamma$ & -0.35 & $\% /{ }^{\circ} \mathrm{C}$ \\
\hline $\mathrm{x}$ & 1 & \\
\hline $\mathrm{T}_{\text {amb }}$ & 25 & ${ }^{\circ} \mathrm{C}$ \\
\hline $\mathrm{y}$ & 0.025 & ${ }^{\circ} \mathrm{C} /\left[\mathrm{W} / \mathrm{m}^{2}\right]$ \\
\hline $\mathrm{T}_{\text {STC }}$ & 25 & ${ }^{\circ} \mathrm{C}$ \\
\hline
\end{tabular}

The values are representative so the results of this analysis are comparable for individual analyses on the different technologies. The irradiation of $135 \mathrm{~W} / \mathrm{m}^{2}$ is a mean value for the coastal region in Belgium [6].

Fig. 9 shows the graphical result of the sensitivity analysis. The percentage change of the instantaneous yield as a function of the percentage change of the formula factors.

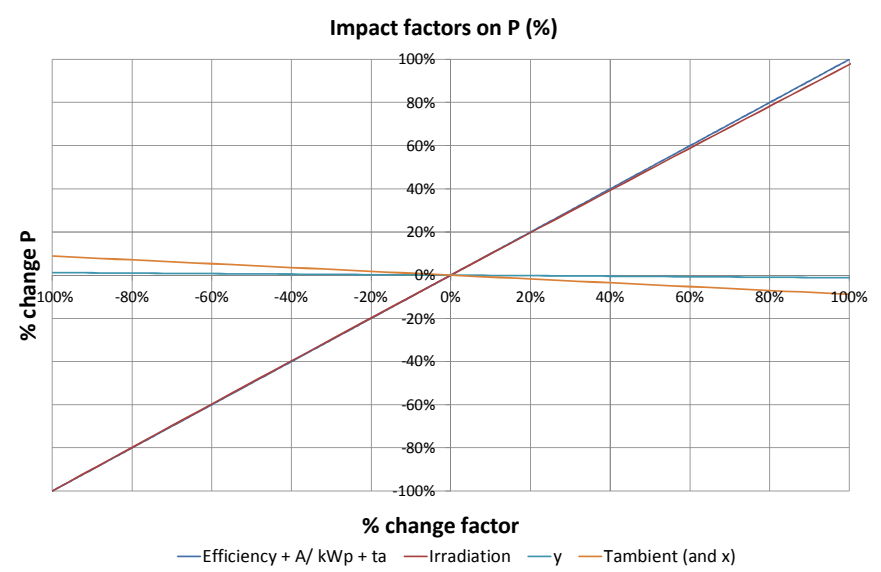

Fig. 9. Sensitivity analysis 
The impact of irradiation is not so clear because of the small mean value of $135 \mathrm{~W} / \mathrm{m}^{2}$ and the $\mathrm{x}$-axis who only gives an increase of $100 \%$. Consequently the impact $y$ of irradiation on module temperature is also not high because of this low constant value of irradiation.

Factors $\eta, \tau \alpha$ and $A_{k W p}$ have an direct proportional impact on the yield. The temperature factors have an inversely proportional impact on the yield.

\section{CONCLUSIONS}

The formula for the prediction of the module temperature uses only two factors. In this study only ambient temperature and irradiation is taken into account. There are more factors with influence such as wind direction, wind temperature and air humidity. It can be concluded that, based on the results, ambient temperature and irradiation are the most important factors. Other factors will lead to a more accurate prediction model but are not required for accurate predictions.

It can be concluded that the combined formula gives an accurate prediction of the module yield except for amorphous technology.

Fig. 10 shows a schematic summary of the used factors in the formula for prediction of instantaneous yield.

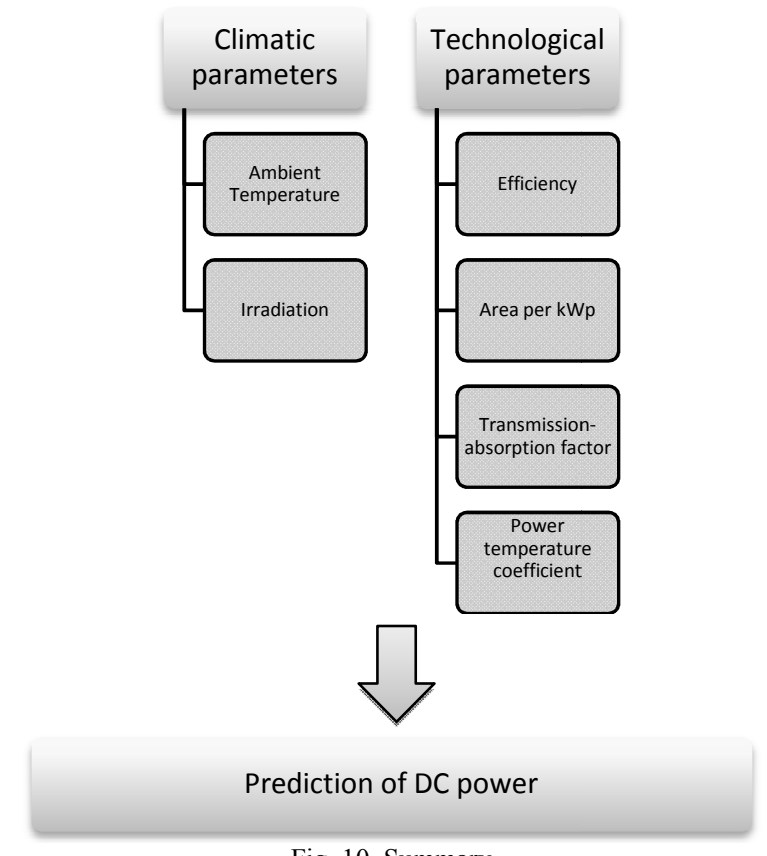

Fig. 10. Summary

The prediction of the instantaneous DC-power of solar panels as a function of technological and climatic parameters is a good representation to the reality. With this formula the impact of each parameter is clarified.

\section{FURTHER RESEARCH}

In future research the equation for the prediction of the instantaneous yield can be refined with some other influence factors, such as degradation of the panel, air humidity, (air-) pollution, shadow, etc. The efficiency of the inverter isn't included in the formula yet, because the system measures DC power.

\section{REMARK ON MEASUREMENT DATA}

December couldn't be taken into account because of the amount of snow days. Snow covers the panel so no irradiation can reach the panel. The device measuring the irradiation is much smaller so snow melts much faster. In this way the predicted values are greater than the actual values and the prediction not credible. If more data from more years is available, a factor expressing the amount of snow days can be determined and added to the formula.

\section{REFERENCES}

[1] H.S. Rauschenbach, Solar Cell Array Design Handbook: The Principles and Technology of Photovoltaic Energy Conversion, United States of America: Van Nostrand Reinhold company, p 52-56/p 168-170

[2] Messenger, R.A., \& Jerry, V. Photovoltaic Systems Engineering, second edition, United States of America: Bocan Raton CRC Press LLC, 2003, p.47-56/p.373-410

[3] E. Skoplaki, J.A. Palyvos, On the temperature dependence of photovoltaic module electrical performance: A review of efficiency/power correlations, Solar Energy, Volume 83, Issue 5, May 2009, Pages 614-624, ISSN 0038-092X, 10.1016/j.solener.2008.10.008.

[4] Hedzlin Zainuddin, Sulaiman Shaari, Ahmad Maliki Omar, Zainazlan Md Zain, Jonson Soumin and zainizam Surat, Preliminary Investigations on the Effect of Humidity on the Reception of Visible Solar Radiation and the Effect of Humidity and Wind speed on PV module Output, AIP Conf. Proc. 1250, pp. 55-58

[5] Govindasamy TamizhMani, Liang Ji, Yingtang Tang and Luis Petacci, "Photovoltaic module thermal/wind performance: Long-term monitoring and model development for energy rating"

[6] Dr. ir. ing. J. Desmet, ing. C. Debruyne, ir. J. Vanalme, ing. B. Verhelst, $\mathrm{BIR}$. Implementatie van innovatieve duurzame energiebronnen en hun interactie op het distributienet. Kortrijk: Howest-Lemcko, 2010, p.35, 39; ISBN 9789081549806

[7] E. Skoplaki, J.A. Palyvos, Operating temperature of photovoltaic modules: A survey of pertinent correlations, Renewable Energy, Volume 34, Issue 1, January 2009, Pages 23-29, ISSN 0960-1481, 10.1016/j.renene.2008.04.009.

[8] Effects of Temperature, Solar Flux and Relative Humidity on the Efficient Conversion of Solar Energy to Electricity, http://www.eurojournals.com/ejsr_35_2_02.pdf

[9] E. Skoplaki, A.G. Boudouvis, J.A. Palyvos, A simple correlation for the operating temperature of photovoltaic modules of arbitrary mounting, Solar Energy Materials and Solar Cells, Volume 92, Issue 11, November 2008, Pages 1393-1402, ISSN 0927-0248, 10.1016/j.solmat.2008.05.016. 